\title{
Earth's Electromagnetic Forces and Their Participation in the Creation of Tornadoes
}

\author{
Yuri Pivovarenko \\ Research and Training Centre 'Physical and Chemical Materials Science, Under Kyiv Taras Shevchenko University and NAS of Ukraine, \\ Kiev, Ukraine
}

\section{Email address: \\ y.pivovarenko@gmail.com}

\section{To cite this article:}

Yuri Pivovarenko. Earth's Electromagnetic Forces and Their Participation in the Creation of Tornadoes. American Journal of Electromagnetics and Applications. Vol. 7, No. 1, 2019, pp. 8-12. doi: 10.11648/j.ajea.20190701.12

Received: September 7, 2019; Accepted: September 27, 2019; Published: October 26, 2019

\begin{abstract}
Both in the earth's atmosphere and on the earth's surface, electromagnetic forces of a understandable nature act. Despite the fact that these forces are quite substantial, their participation in the phenomena occurring on the Earth's surface and in the Earth's atmosphere is traditionally ignored. Such a tradition does not allow explaining the nature of a number of phenomena occurring both in the Earth's atmosphere and on the Earth's surface, for example, the distribution of charges in the direction perpendicular to the Earth's surface, in particular, the distribution of charges existing between the Earth's surface and the upper atmosphere. In particular, such a tradition, thanks to which a tornado is considered solely as a result of mechanical interactions, does not allow us to understand its true nature and, as a result, to suggest ways of its destruction. Since this may have worldview significance, this article is intended to draw attention to electromagnetic forces that act in the Earth's atmosphere and on the Earth's surface.
\end{abstract}

Keywords: Tornado, Creation, Evolution, Tornadogenesis

\section{Introduction}

Attempts to explain the nature of tornadoes, ignoring the participation of electromagnetic forces in its occurrence and evolution, are counterproductive. These are identical to attempts to explain the fall of bodies, ignoring the existence of gravity. So, on the basis of only mechanical forces arising due to the difference in atmospheric pressure [1-3], it is impossible to explain the occurrence of air vortices, as well as a number of other phenomena occurring in the tornado zone [4]. In particular, based solely on mechanical forces, the fact that tornadoes rotate counterclockwise in the northern hemisphere and clockwise in the southern hemisphere cannot be explained. Such a difference in the direction of rotation of the tornado cannot be explained even using the most complete systems of differential equations that take into account exclusively mechanical interactions, but completely ignore the participation of electromagnetic forces in the formation of the tornado $[5,6]$. At the same time, given the opposite directions of the vertical component of the geomagnetic field in the northern and southern hemispheres, this difference in the direction of rotation of the tornado is easy to explain using electromagnetic forces, the nature of which has long been established and is well understood [7]. Thus, using only mechanical ideas, one should not count on progress in understanding the nature of tornadoes, but one will have to ascertain long-term stagnation in this field of knowledge [8].

Thus, in order to achieve progress in understanding the nature of tornadoes, it is necessary to take into account the electromagnetic forces acting both on the earth's surface and in the earth's atmosphere. Apparently, the first of these forces should be called the Lorentz force, which arises due to the daily rotation of the Earth, namely, due to the intersection of the Earth's atmosphere and objects located on the earth's surface, horizontal lines of the geomagnetic field (Figure 1).

Thus, both objects of the Earth's surface and the Earth's atmosphere are constantly exposed to the Lorentz force $\mathrm{F}_{\mathrm{L}}$ *, which is directed exclusively upward and, because of this, moves positive charges upward and negative charges downward. The fact that this force can be very significant is shown by calculations according to which a single proton can 
be accelerated by this force in the upward direction to $4,175 \cdot 10^{7} \mathrm{~m} \cdot \mathrm{s}^{-2}$, and an electron can be accelerated by the same force in the downward direction to $2,7 \cdot 10^{12} \mathrm{~m} \cdot \mathrm{s}^{-2}$, in any case, on the equator line. In addition, the calculations show that, under the action of the same Lorenz force $\mathrm{F}_{\mathrm{L}}{ }^{*}$, a single proton "can lift" a droplet consisting of $\sim 84830$ water molecules $\left(\sim 1,5 \cdot 10^{6} \mathrm{D}\right)$. The last relation is very important because it explains why only positively charged vapor can evaporate $[9,10]$.

Given the influence of this Lorenz force $\mathrm{F}_{\mathrm{L}}{ }^{*}$, it is not surprising that it is able to quickly and efficiently distribute positively and negatively charged vapor in the clouds (Figure 2) $[9]$.

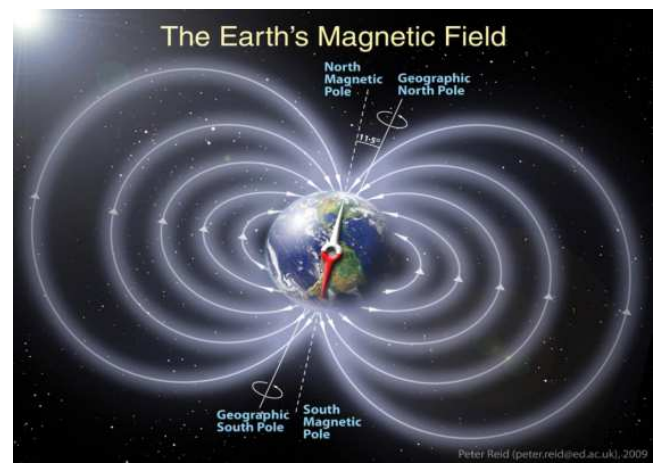

Figure 1. With the Earth's daily rotation, both the Earth's atmosphere and objects located on the Earth's surface constantly cross the lines of force of the geomagnetic field. For this reason, both the Earth's atmosphere and objects located on the Earth's surface are constantly affected by the upward Lorentz force $F_{L}$ *.

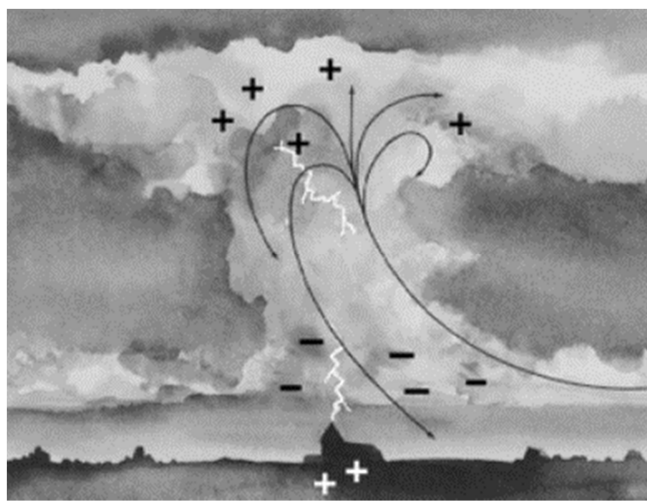

Figure 2. Polarization of clouds: the lower part of a typical cloud has a negative charge and the upper part has a positive charge [9].

Thus, the cloud is formed by charges that are in dynamic equilibrium due to the combined action of the upward Lorentz force $\mathrm{F}_{\mathrm{L}}{ }^{*}$, which separates the charges in the vertical direction, and the downward electrostatic force, which tends to connect them. As well, it is not also surprising that the same Lorenz force $\mathrm{F}_{\mathrm{L}}{ }^{*}$ is able to create the celestial discharges, both up directed positively charged (Figure 3, above), and down directed negatively charged (Figure 3, below) [11].

It is also not surprising that the same Lorentz force $\mathrm{F}_{\mathrm{L}}{ }^{*}$ distributes the flame charges in the same way: the shape of the flame, which is affected by a horizontal electric field, shows that the upper part of the flame is positively charged and its lower part is negatively charged (Figure 4) [12].

All this makes it possible to assume that the same Lorentz force $\mathrm{F}_{\mathrm{L}}{ }^{*}$ causes a positive charge of the upper atmosphere and a negative charge on the Earth's surface. This assumption may be important, given that Richard Feynman wrote that the nature of the force that distributes charges in this way between the surface of the Earth and the ionosphere is unknown [13].
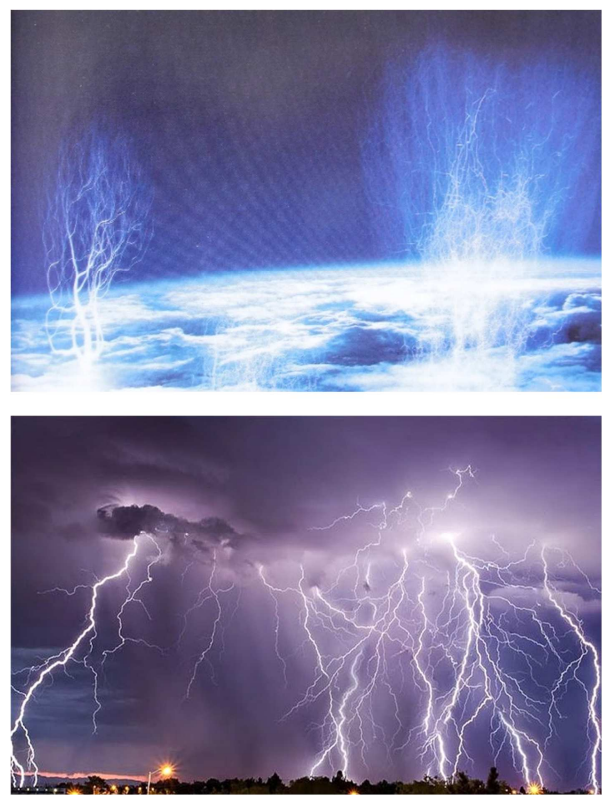

Figure 3. Above: these are blue jets representing ascending currents of hydrated protons. Below: these are ordinary thunderstorms, which are downward flows of hydrated electrons [2].

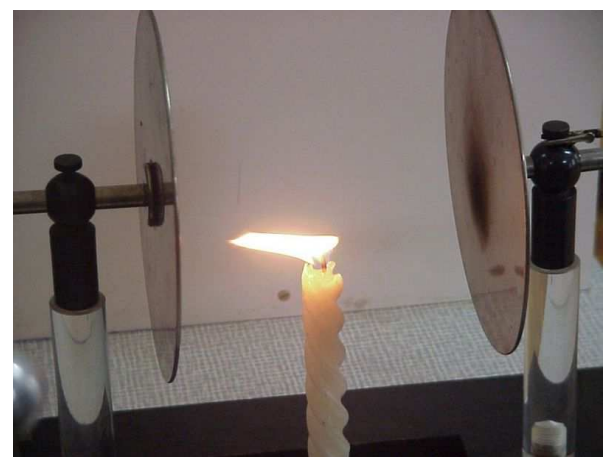

Figure 4. Candle flame under the influence of a left-directed electric field: the upper part of the flame, which is positively charged, deviates to the left, and the lower part of the flame, which is negatively charged, deviates to the right [12].

If we accept that the described Lorentz force $\mathrm{F}_{\mathrm{L}} *$ is indeed influential, it is not surprising that it can increase the lift of positively charged aircraft and reduce the lift of negatively charged aircraft $[12,14]$. And given that a mass of $1.5 \cdot 10^{6} \mathrm{D}$ is comparable to a mass of DNA, it is also not surprising that the protonated DNA molecule can DNA of water into the air and vice versa: in this way, DNA can also be moved between adjacent tubes. This suggests that the "transfer of information from DNA solutions to clean water" (meaning quantum 
teleportation of L. Montaigne) may actually consist in the transfer of DNA molecules.

Thus, it can be recognized that the Lorentz force $\mathrm{F}_{\mathrm{L}}$ * should be taken into account when explaining the nature of terrestrial phenomena, at least some. (In principle, this is not surprising: since this Lorentz force acts on the Earth, there must necessarily be phenomena demonstrating its effect.) At the same time, there are other electromagnetic forces that can influence earthly phenomena. Here I suggest you make sure that such forces participate in the formation of the tornado.

\section{Main Body}

It is clear that all horizontally directed air currents, with the exception of the equatorial ones, invariably cross the vertical component of the geomagnetic field. Thus, each of these air currents is affected by (another force) Lorentz $\mathrm{F}_{\mathrm{L}}{ }^{* *}$, which distributes their electric charges in the horizontal plane and perpendicular to their directions. For this reason, the positive charges are concentrated on one side of any horizontal air flow, and the negative charges on the opposite. (A similar distribution of charges in a metal conductor with a current acting on a magnetic field that is perpendicular to the conductor is called the Hall Effect [6].)

Given that the vertical component of the geomagnetic field in the northern hemisphere is directed downward, the left side of each horizontal air flow, when viewed in its direction, is always charged positively, and its right side is always charged negatively. However, as a result of the action of the (previously described) Lorentz force $\mathrm{F}_{\mathrm{L}}{ }^{*}$, the negatively charged side of any air flow will move downwards, and the positively charged side will move upwards. Due to the simultaneous action of the described Lorentz forces $\left(\mathrm{F}_{\mathrm{L}} *\right.$ and $\mathrm{F}_{\mathrm{L}}{ }^{*}$ ) on horizontal air flows, the trajectories of the positively charged sides of the air flows form ascending spirals twisted counterclockwise, and the trajectories of their negatively charged sides form descending spirals twisted clockwise (of course in the northern hemisphere).

The combined effect of both described Lorentz forces on horizontal air flows can be demonstrated graphically (Figure 5).

I hope that this diagram gives a correct idea of the effect of the described Lorentz forces on horizontal air flows and allows us to understand the basic processes occurring in the tornado zone (Figure 6).

It is clear that this charge separation in the tornado area simultaneously creates a horizontally directed electrostatic field in which the previously separated charges tend to connect again. Thus, a tornado is a system of charges that are in dynamic equilibrium due to the combined action of the electromagnetic forces that seek to separate charges, and an electrostatic force that seeks to connect them, just like in a cloud (Figure 2).

The combined effect of both the described Lorentz forces on horizontal air flows can be graphically demonstrated (Figure 5). I hope that this diagram gives a correct idea of the effect of the described Lorentz forces on horizontal air flows and allows us to understand the basic processes occurring in the tornado area (Figure 6).
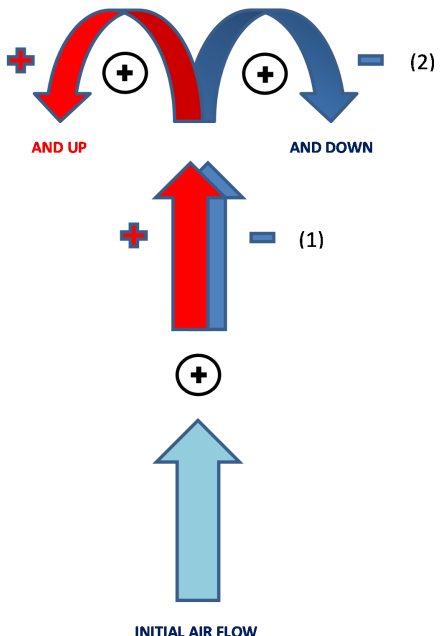

Figure 5. Below is the separation of the horizontal current (blue) when interacting with the downward component of the geomagnetic field, which results in two horizontal currents: positive (red) and negative (dark blue) charged (1). The above shows the further transformation of positively and negatively charged flows under the action of the downward component of the geomagnetic field, namely their rotation counter clockwise (red) and clockwise (dark blue) (2) Due to their interaction with the horizontal component of the geomagnetic field (so as not to complicate the picture, it is not shown here), a positively charged stream moves simultaneously upward, and a negatively charged stream moves simultaneously downward.
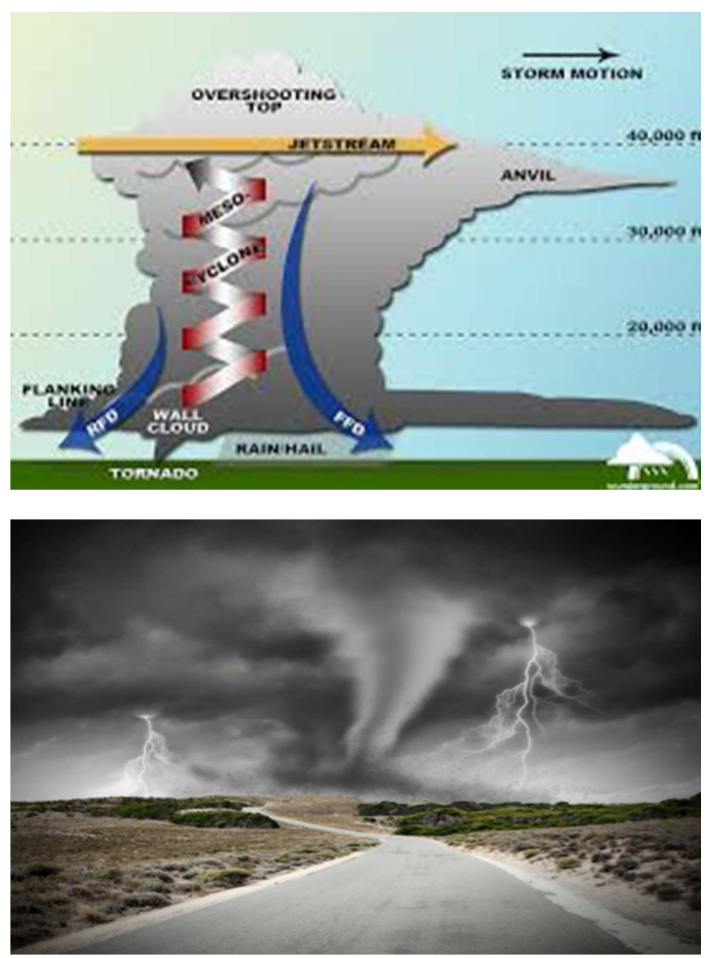

Figure 6. Above: this is a diagram illustrating both the structure of the tornado and the process of its formation: the tornado is formed from a cloud located below the horizontal air flow. Below: A typical tornado is an upward flow of positively charged air that rotates counter clockwise (when viewed from above) and is surrounded by lightning, which are downward flows of negative charges. 
In an effort to observe the effect of these experimentally, we assumed that the negatively charged part of each horizontal air flow can rotate the underlying water more intensively in a clockwise direction than its positively charged part counterclockwise. This assumption was confirmed by the experiment described below. Two waterfilled Petri dishes were placed to the left and right of narrow and horizontal air currents. Agree that it would be quite possible to expect that under the action of such an air flow, the water in these Petri dishes would rotate both clockwise and counterclockwise. At the same time, even with the naked eye it was clear that the water always rotated clockwise very quickly and counterclockwise always very slowly (in fact, in the latter case, the water always remained almost still). Although this difference in rotational speeds can be seen quite well with the naked eye, it is especially convenient to observe it with the help of starch powder (Figure 7).

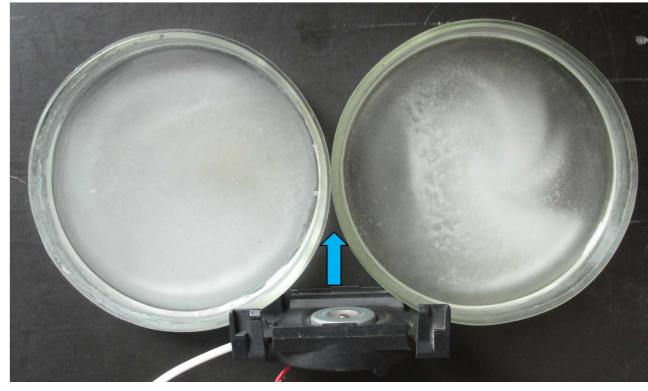

Figure 7. On the left is a Petri dish with an aqueous suspension of starch, which was located to the left and below of the narrow air stream; a precipitate of starch powder, evenly covering the bottom. On the right is a Petri dish with an aqueous suspension of starch, which was located to the right and below of the narrow air flow; the starch powder precipitate formed turbulence. The blue arrow indicates the direction of the air flow from the small cooler.

It should be noted that the intensity of the described rotation can be, to some extent, sensitive to atmospheric pressure, the value of which, as is known, linearly correlates with the electrification of the lower atmosphere [7]. At the same time, such sensitivity confirms the participation of electric forces in the appearance of such rotation.

In addition, direct measurements of the electric potential difference between the solutions of both Petri dishes (Figure 7) showed that the solution from the right Petri dish (Figure 7 , right) always had a negative electric potential relative to the solution from the left Petri dish (Figure 7, left). Thus, our vision of the nature of the processes occurring in the tornado zone has received two experimental confirmations.

Given all this, you can suggest some ways to destroy the tornado. So, if a sufficient negative charge is introduced into the upward flow of a tornado (Figure 6), its positive charge can be reduced or completely neutralized. Thus, the balance between negative and positive charges, due to which a stable tornado structure exists, can be violated, for example, due to the connection of positive and negatively charged parts of the tornado with a metal conductor: in this case, the metal conductor can cause a "short circuit". For the same purpose, one can use the ability of a ray of light to "push" positive charges, i.e. move them in the direction of the Pointing vector, indicating the direction of the light ray [16] (this method of mutual neutralization of the opposite charges of the tornado may be most suitable). On the other hand, the tornado's lifting force can also be significantly reduced by injecting negative charges into its positively charged portion.

To summarize all that has been said, I want to express the hope that my vision of the nature of the tornado will eventually "curb" this formidable phenomenon, and not only endlessly study its features, as is now happening [17-19].

\section{Conclusion}

Powerful electromagnetic forces act both on the Earth's surface and in the Earth's atmosphere, in particular in the tornado zone.

Understanding the features of the emergence of these forces allows you to better understand the nature of some earthly phenomena and makes it possible to control some of them. Thus, an understanding of the electromagnetic nature of tornadoes suggests a fundamental way to destroy its structure or reduce its destructive force.

Based solely on mechanical concepts, it is impossible to understand the true nature of the phenomena that occur on the earth's surface and in the earth's atmosphere.

\section{References}

[1] Church C. R., Snow J. T. (1984) Measurements of Axial Pressures in Tornado-like Vortices. J. Atmos. Sci., 42 (6), 576582.

[2] Pauley R. L. (1989) Laboratory Measurements of Axial Pressures in Two-celled Tornado-like Vortices. J. Atmos. Sci., 46 (21), 3392-3399.

[3] Winn, W. P., Hunyady S. J., Aulich, G. D. (1999) Pressure at the Ground in a Large Tornado. J. Geophys. Res, 104 (18), 22067-22082.

[4] Abdul J. A. (1966) The "Musical" Sound Emitted by a Tornado. Monthly Weather Review, 94 (4), 213-220.

[5] Davies-Jones R. P. (1982) A New Look at the Vorticity Equation with Application to Tornadogenesis. Preprints. 12th Conf. on Severe Local Storms, San Antonio, TX, Amer. Meteor. Soc., 249-252.

[6] Davies-Jones R. P. (1982) Observational and Theoretical Aspects of Tornadogenesis. Intense Atmospheric Vortices. In: L. Bengtsson and J. Lighthill, Eds. Springer-Verlag, 175-189.

[7] Purcell E. (1971) Electricity and Magnetism. BPC, 2. Moscow: Nauka. In Russian.

[8] Edwards R., Ladue J. G., Ferree J. T., Scharfenberg K., Maier C., Coulbourne W. L. (2013) Tornado Intensity Estimation: Past, Present, and Future. Bulletin of the American Meteorological Society, 94 (5), 641-653.

[9] Pivovarenko Y. (2015) A Charge Distribution in the Earth's Atmosphere. American Journal of Physics and Applications, 3 (3), 67-68. 
[10] Krasnogorskaja N. V. (1984) Electromagnetic fields in the earth's atmosphere and their biological significance 1, Moscow: Nauka.

[11] Pivovarenko Y. (2018) The Nature of the Celestial Elves, Sprites and Jets. Discovery Nature, 12, 1-4.

[12] Pivovarenko Y. (2017) The Flight of the Balloon and the Daily Rotation of the Earth. World Journal of Applied Physics, 2 (2), $32-35$.

[13] Feynman R., Leighton R., Sands M. (1971) FLP, 3. Massachusetts: Addison Wesley Publishing Company.

[14] Pivovarenko Y. (2017) The Significance of Positive Electrization on Lifting Force. American Journal of Aerospace Engineering, 4 (6), 59-62.

[15] Kuznetsov V. V., Cherneva N. I., Druzhin G. I. (2007) On the
Influence of Cyclones on the Atmospheric Electric Field of Kamchatka. Reports of the Academy of Sciences, 412 (4), 1-5.

[16] Crawford F. (1974) Waves; BPC, 3. Moscow: Nauka. In Russian.

[17] Bedard A. J. (2005) Low-Frequency Atmospheric Acoustic Energy Associated with Vortices Produced by Thunderstorms. Mon. Weather Rev., 133 (1), 241-263.

[18] Van Den Brooke M. S. (2017) Polarimetric radar metrics related to tornado life cycles and intensity in supercell storms, Mon. Weather Rev., 145, 3671-3686.

[19] Elbing B. R., Petrin C. E. Van Den Brooke M. S. (2019) Measurement and Characterization of Infrasound From a Tornado Producing Storm. The Journal of the Acoustical Society of America, 146, 1528-1537. 